\title{
Identification of responders and reactive domains to rivastigmine in Alzheimer's disease ${ }^{\dagger}$
}

\author{
S. V. Frankfort PharmD ${ }^{1,2 *}$, B. A. Appels $\mathrm{MSc}^{3}$, A. de Boer MD, PhD ${ }^{4}$, L. R. Tulner $\mathrm{MD}^{2}$, \\ J. P. C. M. van Campen $\mathrm{MD}^{2}$, C. H. W. Koks PharmD, $\mathrm{PhD}^{1}$, J. H. Beijnen PharmD, $\mathrm{PhD}^{1,5}$ \\ and B. A. Schmand $\mathrm{PhD}^{6}$ \\ ${ }^{1}$ Department of Pharmacy \& Pharmacology, Slotervaart Hospital, Amsterdam, The Netherlands \\ ${ }^{2}$ Department of Geriatric Medicine, Slotervaart Hospital, Amsterdam, The Netherlands \\ ${ }^{3}$ Department of Medical Psychology, Slotervaart Hospital, Amsterdam, The Netherlands \\ ${ }^{4}$ Department of Pharmacotherapy and Pharmacoepidemiology, Faculty of Pharmaceutical Sciences, Utrecht University, \\ Utrecht, The Netherlands \\ ${ }^{5}$ Department of Biomedical Analysis, Division of Drug Toxicology, Faculty of Pharmaceutical Sciences, Utrecht \\ University, Utrecht, The Netherlands \\ ${ }^{6}$ Department of Neurology, Academic Medical Center, University of Amsterdam, Amsterdam, The Netherlands
}

\section{SUMMARY}

Purpose Presently, it is unclear which patients suffering from Alzheimer's Disease (AD) respond to rivastigmine and if rivastigmine acts on specific cognitive domains. The aims of this study are thus to investigate treatment effects of rivastigmine on specific cognitive domains and to find possible responsive subpopulations to rivastigmine cognitive effects. Methods Mini Mental State Examination (MMSE) and Cambridge Cognitive Examination (CAMCOG) were administered at baseline and after 6 months in 83 rivastigmine users and 96 historical controls, representing natural decline. Treatment effects on different subsections of the CAMCOG and in different subpopulations were investigated by linear regression analyses.

Results Rivastigmine showed effectiveness on total CAMCOG $(p<0.001)$, CAMCOG non-memory subsection $(p<0.001)$ and subscales of language $(p=0.002)$, attention/calculation $(p=0.043)$, abstract thinking $(p<0.001)$ and perception $(p=0.031)$. In patients with baseline MMSE $\leq 19$ rivastigmine showed significant and favourable effects compared to historical controls on total CAMCOG $(p<0.001)$ and both non-memory $(p<0.001)$ and memory subsections $(p=0.002)$.

Conclusion Rivastigmine showed primarily effectiveness on the non-memory section of the CAMCOG and patients with a baseline MMSE $\leq 19$ appeared to show greater responses to rivastigmine compared to patients with baseline MMSE $\geq 20$. Copyright (C) 2006 John Wiley \& Sons, Ltd.

KEY WORDS — rivastigmine; Alzheimer's disease; responders; cognitive domains; CAMCOG

Received 23 May 2006; Revised 20 September 2006; Accepted 3 October 2006

* Correspondence to: S. V. Frankfort, Department of Pharmacy \& Pharmacology, Slotervaart Hospital, Louwesweg 6, 1066 EC Amsterdam, The Netherlands. E-mail: apsfr@slz.nl

${ }^{\dagger}$ No conflict of interest was declared.

Copyright (C) 2006 John Wiley \& Sons, Ltd.

\section{INTRODUCTION}

Rivastigmine, an acetylcholinesterase inhibitor, showed efficacy in the symptomatic treatment of mild-to-moderately severe Alzheimer's disease (AD) in randomised placebo-controlled trials. ${ }^{1,2}$ However, patients respond very differently to therapy, ranging 
from continuation of deterioration or maintaining baseline levels to a clear clinical effect. ${ }^{3}$ Previous research showed improvement in APOE 4 carriers treated with rivastigmine, ${ }^{4}$ more benefit of therapy in patients with a rapid rate of disease progression ${ }^{5,6}$ and an association between the occurrence of hallucinations and response to therapy, as defined by an increase of two or more points on the MMSE. ${ }^{7}$ Starting therapy only in those patients in whom effect is expected would be ideal in clinical practice considering the fact that rivastigmine users often experience adverse events. $^{8}$

It is presently unclear which characteristics identify rivastigmine responders. It is also uncertain if rivastigmine exerts treatment effects on certain cognitive subdomains in $\mathrm{AD}$ as, to our knowledge, no studies regarding this issue have been published. However, attention is one of the cognitive subdomains that indeed responded to rivastigmine therapy in a study performed in Lewy Body Disease (LBD) patients. ${ }^{9}$ Therefore, attention might also specifically respond to therapy in AD patients.

The aims of this study are to investigate treatment effects of rivastigmine on specific cognitive domains and to find possible responsive subpopulations to rivastigmine cognitive effects.

\section{METHODS}

\section{Patients}

This prospective study was carried out in patients with mild-to-moderate, probable or possible AD according to the NINCDS-ADRDA criteria. ${ }^{10}$ The index group consisted of patients using rivastigmine $\left(\right.$ Exelon $^{\mathbb{R}}$ ) via the geriatric outpatient department of a Dutch hospital. Only patients who had relatives or friends who could monitor drug intake and patients in whom therapy was evaluated after 6 months were included. The historical control cohort consisted of Alzheimer patients who did not take rivastigmine and were followed during a period of 6 months as part of a research project regarding the utility of diagnostics procedures in a memory clinic in The Netherlands. ${ }^{11}$ This historical control group represents natural decline in $\mathrm{AD}$. Patients were excluded if cognitive test results were incomplete. Education was scored on a seven-point scale, ranging from less than 6 years of elementary school (1) to a university degree (7). ${ }^{12}$

The review board of the Slotervaart Hospital, Amsterdam, The Netherlands, approved the use of routine anonymous neuropsychological assessment data for research purposes.

\section{Assessment of domains}

At baseline, that is when starting rivastigmine in case of the index group, and after 6 months, patients were evaluated by cognitive assessment including Mini Mental State Examination (MMSE; max score: 30$)^{13}$ and Cambridge Cognitive Examination (CAMCOG) ${ }^{14}$ CAMCOG consists of 60 items and total sum scores range from 0 to 107. CAMCOG can be subdivided into a memory (max score: 37 ) and a non-memory (max score: 70) section. ${ }^{15}$ The memory subsection can be further subdivided into subscales regarding recent memory ( $\max$ score: 4), remote memory (max score: 6), learning (max score: 17) and orientation ( $\max$ score: 10). The non-memory subsection can be subdivided into subscales assessing language (max score: 30), praxis (max score: 12), attention/calculation (max score: 9), abstract thinking (max score: 8 ) and perception (max score: 11). One item in the CAMCOG perception subscale (asking if the respondent recognised two people in the room) was omitted and always scored as one point. Lower scores on MMSE and CAMCOG reflect more severe disease.

\section{Statistical analysis}

(A) We performed linear regression analyses to investigate rivastigmine effectiveness on cognition compared to the historical control cohort during 6 months as measured by MMSE, CAMCOG and subsections and subscales of the CAMCOG. The dependent variable was the test result after 6 months and the independent variable was rivastigmine use.

(B) We investigated whether effect modification plays a role by introducing interaction terms in linear regression analyses regarding CAMCOG total, non-memory and memory subsections and the attention subscale. Therefore, we sequentially performed multivariate linear regression analyses including the interaction term rivastigmine by dichotomised disease severity, in addition to both terms separately. As an indicator for disease severity, the baseline MMSE score was dichotomised to the median of the whole population, resulting in MMSE $\leq 19$ versus $\geq 20$. If the interaction terms showed significance, we performed additional linear regression analyses to investigate rivastigmine effects in subgroups. We corrected analyses for age, gender and level of education, as these variables may influence cognitive performance, and for baseline test results. All statistical 
Table 1. Baseline characteristics

\begin{tabular}{lcc}
\hline Baseline characteristics/test results & Rivastigmine users $(n=83)^{*}$ & Historical controls $(n=96)^{*}$ \\
\hline Age (years) & $78.1 \pm 6.0(56-89)$ & $77.8 \pm 5.9(65-89)$ \\
Education, median, IQR (range) & 4.0, IQR $3.0(1-6)$ & 2.0, IQR $2.0(1-7)$ \\
Female gender, $n$ (\%) & $57(68.7)$ & $55(57.3)$ \\
MMSE & $20.5 \pm 4.3(8-28)$ & $18.4 \pm 5.2(1-28)$ \\
CAMCOG total & $69.0 \pm 13.0(33-92)$ & $62.8 \pm 16.0(12-94)$ \\
CAMCOG memory & $17.5 \pm 5.5(5-29)$ & $16.6 \pm 7.6(0-32)$ \\
Orientation & $6.6 \pm 2.1(2-10)$ & $6.0 \pm 2.5(0-10)$ \\
Recent memory & $1.8 \pm 1.1(0-4)$ & $1.6 \pm 1.3(0-4)$ \\
Remote memory & $3.0 \pm 1.7(0-6)$ & $2.7 \pm 1.9(0-6)$ \\
Learning & $6.1 \pm 2.6(0-11)$ & $6.2 \pm 3.5(0-14)$ \\
CAMCOG non-memory & $51.6 \pm 9.3(25-67)$ & $46.3 \pm 10.1(12-64)$ \\
Language & $23.1 \pm 3.5(12-29)$ & $21.4 \pm 4.1(7-28)$ \\
Calculation/attention & $6.7 \pm 2.4(1-9)$ & $5.7 \pm 2.2(1-9)$ \\
Praxis & $9.4 \pm 2.0(4-12)$ & $7.9 \pm 2.5(1-12)$ \\
Abstract thinking & $5.1 \pm 2.3(0-8)$ & $3.8 \pm 2.3(0-8)$ \\
Perception & $7.3 \pm 2.4(1-11)$ & $7.5 \pm 1.8(3-10)$ \\
\hline
\end{tabular}

IQR, inter quartile range.

${ }^{*}$ Expressed as Mean \pm SD (range), unless otherwise stated.

calculations were performed with SPSS for Windows (version 11.0, SPSS Inc., Chicago, IL).

\section{RESULTS}

\section{Patient characteristics}

In total, 179 patients were included in the study of whom 83 used rivastigmine during 6 months and 96 were historical control patients with complete cognitive screening results. Twenty-four patients were excluded from the historical control cohort and one patient was excluded from the index group because cognitive test results were incomplete. Table 1 shows baseline characteristics and baseline test results of included patients. Median baseline MMSE score of all included patients was 20 (IQR: 7.00, range 1-28), median age was 79.0 (IQR: 8.00, range 56-89), almost $63 \%$ were women and the median education level was 3 (IQR: 3.00 , range $1-7$ ).

\section{Rivastigmine effectiveness during 6 months}

Table 2 presents the results of the linear regression analyses investigating if rivastigmine use exerts significant effects as measured by MMSE, CAMCOG total score and subscales assessing specific cognitive domains and adjusted for baseline cognitive test results, age, gender and level of education. Rivastigmine use showed a significant effect on MMSE (Mean difference compared to controls $(\mathrm{MD}=1.7)$ ), CAMCOG total score $(\mathrm{MD}=4.4)$ and the nonmemory section of this instrument $(\mathrm{MD}=3.5)$. On
Table 2. Treatment effects of rivastigmine as measured on MMSE, CAMCOG total score, subsections and subscales assessing specific cognitive domains and expressed as corrected mean differences from linear regression analyses

\begin{tabular}{lcrr}
\hline (Sub) test & \multicolumn{3}{c}{ Linear regression analyses* } \\
\cline { 2 - 4 } & $\begin{array}{c}\text { Mean } \\
\text { difference }\end{array}$ & $95 \%$ CI & $p$-value \\
\hline MMSE & 1.7 & $0.7-2.6$ & 0.001 \\
CAMCOG total & 4.4 & $2.3-6.4$ & $<0.001$ \\
CAMCOG non-memory & 3.5 & $1.9-5.2$ & $<0.001$ \\
Language & 1.5 & $0.6-2.4$ & 0.002 \\
Attention/calculation & 0.6 & $0.0-1.1$ & 0.043 \\
Praxis & 0.3 & $-0.3-0.8$ & 0.333 \\
Abstract thinking & 1.3 & $0.8-1.9$ & $<0.001$ \\
Perception & 0.5 & $0.0-1.0$ & 0.031 \\
CAMCOG memory & 0.8 & $-0.2-1.8$ & 0.108 \\
Memory: recent & 0.1 & $-0.2-0.4$ & 0.565 \\
Memory: remote & 0.1 & $-0.2-0.5$ & 0.568 \\
Memory: learning & 0.5 & $-0.2-1.1$ & 0.170 \\
Orientation & 0.4 & $-0.2-1.0$ & 0.152 \\
\hline
\end{tabular}

CI, confidence interval.

${ }^{*}$ Corrected for age, gender, education level and baseline scores.

the contrary, no significant effects on the scores of the memory section were shown. By investigating subscales of the non-memory subsection, rivastigmine did show significant effects on language $(\mathrm{MD}=1.5)$, attention/calculation ( $\mathrm{MD}=0.6)$, abstract thinking $(\mathrm{MD}=1.3)$ and perception $(\mathrm{MD}=0.5)$. Praxis, orientation and subscales of remote, recent and learning memory did not show significant effects of rivastigmine use. 
Table 3. Differential treatment effects of rivastigmine in subpopulations expressed as mean differences from linear regression analysis corrected for age, gender, education level and baseline scores

\begin{tabular}{|c|c|c|c|c|}
\hline \multirow[t]{2}{*}{ Test } & \multirow[t]{2}{*}{ Subpopulation } & \multicolumn{3}{|c|}{ Linear regression analysis* } \\
\hline & & Mean difference & $95 \% \mathrm{CI}$ & $p$-value \\
\hline \multirow[t]{2}{*}{ CAMCOG total } & $\mathrm{MMSE} \leq 19$ & 8.2 & $5.0-11.3$ & $<0.001$ \\
\hline & MMSE $\geq 20$ & 0.7 & $-1.9-3.3$ & 0.582 \\
\hline \multirow[t]{2}{*}{ CAMCOG memory } & $\mathrm{MMSE} \leq 19$ & 2.2 & $0.8-3.5$ & 0.002 \\
\hline & MMSE $\geq 20$ & -0.5 & $-2.0-1.0$ & 0.534 \\
\hline \multirow[t]{2}{*}{ CAMCOG non-memory } & $\mathrm{MMSE} \leq 19$ & 6.3 & $3.7-9.0$ & $<0.001$ \\
\hline & MMSE $\geq 20$ & 0.6 & $-1.4-2.6$ & 0.531 \\
\hline \multirow[t]{2}{*}{ Attention/calculation } & MMSE $\leq 19$ & 1.2 & $0.4-2.0$ & 0.05 \\
\hline & $\mathrm{MMSE} \geq 20$ & 0.2 & $-0.5-0.9$ & 0.596 \\
\hline
\end{tabular}

CI, confidence interval.

${ }^{*}$ Corrected for age, gender, education level and baseline scores.

\section{Predictors of rivastigmine effectiveness}

Only the interaction terms that showed significance in different analyses are presented in this section. The interaction term of rivastigmine by disease severity, indicating differential responses to rivastigmine in patients with baseline MMSE $\leq 19$ compared to MMSE $\geq 20$ showed significance on CAMCOG total $(p=<0.001)$, CAMCOG-non-memory $(p=0.001)$, attention/calculation $(p=0.003)$ and CAMCOG memory $(p=0.004)$.

\section{Differential effectiveness in subpopulations}

Table 3 shows treatment effects in subpopulations as defined by significant interaction terms as described above. It is clear from the table that the described subpopulations showed differential rivastigmine effectiveness, concerning both statistically significant effects as well as size of the treatment effect expressed as the mean difference (MD) of test scores in the index group compared to the reference group. Disease severity (MMSE $\leq 19$ ) is an important identifying variable as in the studied population rivastigmine showed significant improvement compared to historical controls on total CAMCOG $(\mathrm{MD}=8.2)$, nonmemory subsection $(\mathrm{MD}=6.3)$, attention/calculation $(\mathrm{MD}=1.2)$ and the memory subsection $(\mathrm{MD}=2.2)$. The described results mean that patients with more severe $\mathrm{AD}$ respond better to rivastigmine.

\section{DISCUSSION}

Rivastigmine shows favourable cognitive effects as measured by MMSE, total CAMCOG and the total score on the non-memory subsection of this instru- ment. The non-memory subscales of language, attention/calculation, abstract thinking and perception also showed improvement as compared to a historical control cohort of Alzheimer patients. However, total scores on the memory section and all subscales of memory, including orientation, as well as the non-memory subscale of praxis did not show significant favourable rivastigmine effects as compared to historical control patients.

Baseline MMSE score can identify a subpopulation of responders to rivastigmine. Patients with a baseline MMSE score $\leq 19$ show significant and favourable effects as compared to historical controls on MMSE, total CAMCOG, memory and non-memory subsection scores and the cognitive subscale of attention/ calculation. Patients with a baseline MMSE $\geq 20$, however, do not show significant effectiveness on these domains in this clinically-based study.

A review ${ }^{16}$ criticised the methodology of cholinesterase inhibitor trials performed during the last years and questioned whether small clinical effects and insufficient methodology are enough scientific basis for prescribing these drugs in AD. However, in our study, we did show clinical effects, as measured by CAMCOG and these effects were more pronounced in different subpopulations. For example, in the subpopulation of more severe disease, our results show a MD compared to control patients of eight points on total CAMCOG scores, which is regarded as a large difference in clinical practice. Our study also confirms the hypothesis of differential efficacy in cholinesterase inhibitor users. ${ }^{3}$

Our results show cognitive subdomains to respond differently to rivastigmine. Attention responds significantly and favourably and may possibly play a mediating role in effectiveness in other cognitive 
domains. Acetylcholine plays an important role in attentional processing and cholinergic dysfunction interferes probably indirectly with cognitive functioning via attention. ${ }^{17}$ A study by Sahakian and Coull ${ }^{18}$ showed that tacrine improved attentional functions in patients with $\mathrm{AD}$. In addition, rivastigmine studies performed in LBD patients, where the cholinergic deficit is probably even larger than in $\mathrm{AD},{ }^{19}$ showed effectiveness on attention. ${ }^{9}$ To our knowledge, this is the first study reporting rivastigmine differential effects in AD. However, in a trial with tacrine, a first generation cholinesterase inhibitor, patients responded significantly better compared to those receiving placebo on Alzheimer's disease Assessment Scale-Cognition (ADAS-Cog) section items assessing recall, naming, language and word finding. ${ }^{20,21}$ The differential effectiveness on cognitive domains, as our investigations showed, should be most ideally confirmed by placebo-controlled trials and/or by research using tests that are more specific.

Previous research investigated rivastigmine use in more advanced $\mathrm{AD}^{22,23}$ Burns et al. ${ }^{23}$ suggested subjects with more severe disease might also benefit from rivastigmine and Kurz et al. ${ }^{22}$ showed cognitive benefits to be more marked in moderate and moderately severe cohorts than the mild AD cohort. For the cholinesterase inhibitor galantamine, it was observed that patients with MMSE scores lower than 18 had a more robust response to therapy ${ }^{24}$ and similarly for another cholinesterase inhibitor, donepezil, a more robust response was noticed in patients with MMSE scores of 20 or below. ${ }^{25}$ Recently, the National Institute for Health and Clinical Excellence, $\mathrm{UK}$, (NICE) has issued a draft of a revised guideline that recommends donepezil, galantamine or rivastigmine to be considered only in the treatment of persons with AD of moderate severity only (MMSE 10-20). ${ }^{26}$ It is clear that disease severity may serve as a variable in identifying responsive subpopulations. A possible explanation could be a larger cholinergic deficit in more severe stages of the disease ${ }^{27}$ suggesting this subpopulation to be more responsive to cholinergic enhancement. As attention may play a key role in cognitive functioning ${ }^{17}$ and differential attentional deficits were shown in different stages of $A D,{ }^{28}$ this may also attribute to a possible explanation why patients with MMSE $\leq 19$ respond significantly better to rivastigmine therapy. Perry et al. ${ }^{28}$ investigated sustained, divided and selective attention in different severity stadia of AD. In early AD (MMSE 24-30), only selective attention showed deficits, while patients with moderately severe AD (MMSE 18-23) showed impairment in sustained, divided and selective

Copyright (C) 2006 John Wiley \& Sons, Ltd. attention. Finally, the rate of disease progression, as measured on scales, does not show linearity during the course of AD. A more marked change occurred in the moderate range. ${ }^{29-31}$ Farlow et al. ${ }^{5}$ showed a more rapid rate of disease progression to be a predictor of rivastigmine effectiveness. This could possibly attribute to our results showing more effectiveness in more severe AD.

CAMCOG evaluates a broad range of cognitive functions that are often affected in dementia. ${ }^{14}$ Although previous research showed this instruments' utilities for assessing and monitoring cognitive decline in moderate and moderately severe patients ${ }^{32}$ and reliability of individual subscales has been reported as acceptable, ${ }^{33}$ one may question the use of the CAMCOG as a monitoring instrument. Lindeboom et al. ${ }^{34}$ showed the CAMCOG to be a non-linear scale. Thus, observed score differences may possibly not represent equal differences in changes in cognition across the test's range. In addition, it is important to keep in mind possible floor and ceiling effects of cognitive tests, which could influence the less marked changes in early and late disease. Ceiling effects, however, are not supposed when using CAMCOG for cognitive evaluation in $\mathrm{AD}$, as it showed little ceiling effect when used in the non-demented elderly ${ }^{35}$ and appeared to be sensitive to the early stages of dementia. $^{36}$ Possible floor effects of CAMCOG screening are in our investigations of minor importance as we included few patients with low baseline CAMCOG scores.

However, these results should be interpreted with caution. We did not conduct a randomised clinical trial. Our study was designed in clinical practice and included patients treated with rivastigmine and historical control patients who were untreated. Bias may have been introduced by this study design. The historical control cohort, for example, was evaluated years before the index group. As dementia care has changed over the years, this may be a confounder in our analysis. Therefore, our results should be

\section{KEY POINTS}

- Rivastigmine effectiveness is more pronounced on non-memory cognitive subdomains.

- Disease severity can be used to identify responsive subpopulations to rivastigmine.

- More severe Alzheimer's disease, defined as baseline MMSE $\leq 19$, is an important subpopulation showing effectiveness of rivastigmine.

Pharmacoepidemiology and Drug Safety, 2007; 16: 545-551

DOI: $10.1002 / p d s$ 
confirmed preferably by prospective randomised controlled trials. The Investigation in the Delay to Diagnosis of AD with Exelon (InDDEx) study is currently conducted and will provide us with knowledge regarding efficacy of rivastigmine in Mild Cognitive Impairment. ${ }^{37}$

\section{CONCLUSION}

Rivastigmine showed differential effectiveness on cognitive subdomains that is more pronounced on the non-memory subsection. In addition, disease severity can be used to identify responsive subpopulations. More severe AD, as scored by a baseline MMSE $\leq 19$, appeared to be an important subpopulation showing more effectiveness to rivastigmine compared to patients with baseline MMSE $\geq 20$.

\section{ACKNOWLEDGEMENTS}

The authors thank Gerard Walstra, neurologist and Saskia Teunisse, neuropsychologist, for their permission to use the data of the historical control cohort in the described study.

\section{REFERENCES}

1. Corey-Bloom J, Anand R, Veach J. A randomized trial evaluating the efficacy and safety of ENA 713 (rivastigmine tartrate), a new acetylcholinesterase inhibitor, in patients with mild to moderately severe Alzheimer's disease. Int J Geriatr Psychopharmacol 1998; 1: 55-65.

2. Rösler M, Anand R, Cicin-Sain A, et al. Efficacy and safety of rivastigmine in patients with Alzheimer's disease: international randomised controlled trial. Br Med J 1999; 318: 633-638.

3. Rockwood K, MacKnight C. Assessing the clinical importance of statistically significant improvement in anti-dementia drug trials. Neuroepidemiology 2001; 20: 51-56.

4. Farlow MR, Lane R, Kudaravalli S, He Y. Differential qualitative responses to rivastigmine in APOE 4 carriers and noncarriers. Pharmacogenomics $J$ 2004; 4: 332-335.

5. Farlow MR, Hake A, Messina J, et al. Response of patients with Alzheimer's disease to rivastigmine treatment is predicted by the rate of disease progression. Arch Neurol 2001; 58: 417-422.

6. Farlow MR, Small GW, Quarg P, Krause A. Efficacy of rivastigmine in Alzheimer's disease patients with rapid disease progression: results of a meta-analysis. Dement Geriatr Cogn Disord 2005; 20: 192-197.

7. Pakrasi S, Mukaetova-Ladinska EB, McKeith IG, O'Brien JT. Clinical predictors of response to acetyl cholinesterase inhibitors: experience from routine clinical use in Newcastle. Int $J$ Geriatr Psychiatry 2003; 18: 879-886.

8. Gauthier S. Cholinergic adverse events of cholinesterase inhibitors in Alzheimer's disease. Epidemiology and management. Drugs Aging 2001; 18: 853-862.

9. McKeith I, Del Ser T, Spano P, et al. Efficacy of rivastigmine in dementia with Lewy Bodies: a randomized, double-blind, placebo-controlled international study. Lancet 2000; 356: 2031-2036.

10. McKhann G, Drachman D, Folstein M, et al. Clinical diagnosis of Alzheimer's disease: report of the NINCDS-ADRDA work group under the auspices of Department of Health and Human Services Task Force on Alzheimer's disease. Neurology 1984; 34: 939-944.

11. Walstra GJ, Teunisse S, van Gool WA, van Crevel H. Reversible dementia in elderly patients referred to a memory clinic. J Neurol 1997; 244: 17-22.

12. Verhage F. Intelligence and Age. (in Dutch). van Gorcum: Assen. 1964.

13. Folstein MF, Folstein SE, McHugh PR. "Mini-mental state". A practical method for grading the cognitive state of patients for the clinician. J Psychiatr Res 1975; 12: 189-198.

14. Roth M, Tym E, Mountjoy CQ, et al. CAMDEX. A standardised instrument for the diagnosis of mental disorder in the elderly with special reference to early detection of dementia. $\mathrm{Br} J$ Psychiatry 1986; 149: 698-709.

15. Schmand B, Walstra G, Lindeboom J, et al. Early detection of Alzheimer's disease using the Cambridge Cognitive Examination (CAMCOG). Psychol Med 2000; 30: 619-627.

16. Kaduszkiewicz H, Zimmermann T, Beck-Bornholdt HP, van den Bussche $\mathrm{H}$. Cholinesterase inhibitors for patients with Alzheimer's disease: systematic review of randomised clinical trials. Br Med J 2005; 331: 321-323.

17. Francis PT, Palmer AM, Snape M, Wilcock G. The cholinergic hypothesis of Alzheimer's disease: a review of progress. J Neurol Neurosurg Psychiatry 1999; 66: 137-147.

18. Sahakian BJ, Coall JT. Nicotine and tetrahydroaminoacradine: evidence for improved attention in patients with dementia of the Alzheimer type. Drug Dev Res 1994; 31: 80-88.

19. Tiraboschi P, Hansen LA, Alford M, et al. Cholinergic dysfunction in diseases with Lewy bodies. Neurology 2000; 54: 407-411.

20. Farlow M, Gracon SI, Hershey LA, et al. A controlled trial of tacrine in Alzheimer's disease. JAMA 1992; 268: 2523-2529.

21. Cummings JL. Use of cholinesterase inhibitors in clinical practice: evidence-based recommendations. Am J Geriatr Psychiatry 2003; 11: 131-145.

22. Kurz A, Farlow M, Quarg P, Spiegel R. Disease stage in Alzheimer's disease and treatment effects of rivastigmine. Alzheimer Dis Assoc Disord 2004; 18: 123-128.

23. Burns A, Spiegel R, Quarg P. Efficacy of rivastigmine in subjects with moderately severe Alzheimer's disease. Int $J$ Geriatr Psychiatry 2004; 19: 243-249.

24. Wilcock GK, Lilienfeld S, Gaens E. Efficacy and safety of galantamine in patients with mild to moderate Alzheimer's disease: multicentre randomised controlled trial. $\mathrm{Br} \mathrm{Med} \mathrm{J}$ 2000; 321: 1445-1449.

25. McLendon B, Murali Doraiswamy P. Defining meaningful change in Alzheimer's disease trials: the donepezil experience. J Geriatr Psychiatry Neurol 1999; 12: 39-48.

26. NICE 2006/001: NICE consults on revised first draft guidance on the use of drugs to treat Alzheimer's disease. http://www. nice.org.uk/pdf2006_001_Alz_Press_release_AD_App_Jan06. pdf. Accessed March 28, 2006.

27. Davis KL, Mohs RC, Marin D, et al. Cholinergic markers in elderly patients with early signs of Alzheimer's disease. JAMA 1999; 281: 1401-1406.

28. Perry RJ, Watson P, Hodges JR. The nature and staging of attention dysfunction in early (minimal and mild) Alzheimer's disease: relationship to episodic and semantic memory impairment. Neuropsychologica 2000; 38: 252-271. 
29. Morris JC, Edland S, Clarck C, et al. The consortium to establish a registry for Alzheimer's disease (CERAD). Part IV: rates of cognitive change in the longitudinal assessment of probable Alzheimer's disease. Neurology 1993; 43: 2457-2465.

30. Stern Y, Liu X, Albert M, et al. Application of a growth curve approach to modeling the progression of Alzheimer's disease. J Gerontol 1996; 51A: M179-M184.

31. Mendiondo MS, Wesson Ashford J, Kryscio RJ, Schmitt FA. Modelling mini-mental state examination changes in Alzheimer's disease. Stat Med 2000; 19: 1607-1616.

32. Garre-Olmo J, López-Pousa S, Vilalta-Franch J, et al. Neuropsychological profile of Alzheimer's disease in women: moderate and moderately severe cognitive decline. Arch Womens Ment Health 2004; 7: 27-36.

33. Cullum S, Huppert FA, McGee M, et al. Decline across different domains of cognitive function in normal ageing: results of a longitudinal population-based study using CAMCOG. Int $J$ Geriatr Psychiatry 2000; 15: 853-862.

34. Lindeboom R, Schmand B, Holman R, et al. Improved brief assessment of cognition in aging and dementia. Neurology 2004; 63: 543-546.

35. Huppert FA, Brayne C, Gill C, et al. CAMCOG-a concise neuropsychological test to assist dementia diagnosis: sociodemographic determinants in an elderly population sample. Br J Clin Psychol 1995; 34: 529-541.

36. Williams JG, Huppert FA, Matthews FE, Nickson J. Performance and normative values of a concise neuropsychological test (CAMCOG) in an elderly population sample. Int $J$ Geriatr Psychiatry 2003; 18: 631-644.

37. Farlow MR, He Y, Tekin S, et al. Impact of APOE in mild cognitive impairment. Neurology 2004; 63: 18981901. 